\title{
ANALYTICAL MODELS OF UNSTABLE RAREFIED GAS FLOW IN CHANNELS AND NOZZLES: SIMULATION AND COMPARISON
}

\author{
O. A. Aksenova and I. A. Khalidov \\ Peter the Great St. Petersburg Polytechnical University, Department of \\ Mathematics \\ 29 Polytechnicheskaya Str., St. Petersburg 195251, Russia
}

\begin{abstract}
Three different analytical models of unstable rarefied gas flows in channels and nozzles are compared using numerical simulations by MonteCarlo method. Numerical results have demonstrated the chaotic behavior of constructed nonlinear dynamic system and the limit properties of corresponding trajectories, attractors, and bifurcations of rarefied gas flows in channels. Flow conditions satisfying the experimental requirements are indicated where the instability of considered type can be detected. The advantages and the drawbacks of the considered approximations are detected and the features of obtained solutions are indicated. Recommendations are given for applying the results in practical applications and in numerical calculations of rarefied gas flows.
\end{abstract}

\section{INTRODUCTION}

The methods of nonlinear dynamics are applied first by Miroshin to study rarefied gas flows in channels [1-3]. Considering the trajectory of each gas atom as a sequence of the reflections from the channel walls (according to the assumption that gas flow is free-molecular), the limit trajectories (i. e., attractors) by increasing number of collisions with the surface have been found analytically. Nonlinear dynamic system is studied corresponding to the multiple scattering of rarefied gas particles from the walls of a channel or of a nozzle.

The authors' previous analytical and numerical investigations have shown that the limit behavior of the considered system is not chaotic everywhere [4$7]$, but there are regions of instability only in a subset of the phase space. In usual applications of the chaos theory, the most interesting cases arise when the chaotic behavior takes place on a "strange attractor." Around this attractor, a large set of initial conditions will lead to orbits that converge to this chaotic

(C) The Authors, published by EDP Sciences. This is an open access article distributed under the terms of the Creative Commons Attribution License 4.0 (http://creativecommons.org/licenses/by/4.0/). 
region. However, in a rarefied gas flow, the most interesting thing is not a strange attractor which can be observed in rarefied gas flows but a cascade of bifurcations [1-8]. The transition from regular to chaotic behavior of the system is here especially interesting, because such transition indicates the values of the parameters where the solution changes substantially with a very small deviation of these parameters.

Rarefied gas flows are discrete dynamic systems (unlike continuous dynamic systems specified by differential equations), because they are described by the laws of mutual interactions of gas particles and of collisions with the surface. Thus, these systems can exhibit strange attractors whatever their dimensionality, whereas in a continuous dynamical system according to the Poincaré-Bendixson theorem, a strange attractor can only arise if the system has three or more dimensions. The cascade of bifurcations appearing for the ray model of the scattering function $V$ was considered in [5]. The ray model, as well as the specular model, determines only one velocity of reflected gas atoms by given incident velocity. However, the angles of incident and of reflected gas atoms for the ray model could be different. The advantages of the ray model are various analytical expressions of momentum exchange coefficients (depending on the angles of incidence and of reflection) and the variety of the shapes of limit gas atoms trajectories. The diffuse addition (multiplied by the coefficient $\sigma$ ) to the ray model gives us the more general ray-diffuse model. This addition causes a randomization and changes fundamentally the limit behavior of the studied dynamic system $[6,7]$.

The main purpose of these new calculations is the analytical and numerical investigation of the properties of the nonlinear dynamic system corresponding to a rarefied gas flow not only for different values of the parameters, but also for different analytical approximations of momentum exchange coefficients. From the point of view of nonlinear dynamics, it corresponds to different iterative equations describing the trajectory of a gas atom. Basic assumption is high Knudsen number $\mathrm{Kn}>1$ (the number of mutual gas atoms interactions must be much less than the number of gas-surface collisions). Applying local interaction approximations allows taking into account that Knudsen number is finite, because the local interaction theory, being exact for free-molecular flow, is confirmed by experimental approximations of momentum and energy exchange coefficients for transition regime between free-molecular and continuum flows. Meanwhile, the bifurcations, the attractors, and the corresponding physical values in the flows have been detected. However, the problem of the empirical confirmation of the obtained numerically effect is still difficult, because the scattering conditions, as well as the regions of the parameters, are quite particular. Therefore, they are hardly reproducible experimentally. The considered bifurcations can essentially affect different gas flows applied in practice such as flows in propulsion systems and in microelectronic vacuum devices. 
The parameters of rarefied gas flow in a channel are naturally connected with the parameters of the scattering function $V\left(\vec{u}, \vec{u}^{\prime}\right)$. This function $V$ depends on the velocities $\vec{u}$ of incident upon the channel wall and $\vec{u}^{\prime}$ of reflected from the surface gas atoms. The scattering function $V$ determines entirely the parameters of a rarefied gas flow in a channel (if the geometric shape is known) at high Knudsen number $\mathrm{Kn}$ (near free-molecular flow, $\mathrm{Kn} \rightarrow \infty$ ). There is no need to eliminate possible mutual collisions of gas atoms (i. e., to assume free-molecular flow), because these collisions are taken into account by applying local approximations [4]. However, the solution cannot be extended to the whole region of Kn because according to the locality hypothesis, most of the incident gas atoms must come from channel walls, so that the influence of mutual collisions of gas atoms is small [4].

In the previous analytical and numerical investigations [4-8], the instability of the flow is detected in long enough channels or nozzles for some transition parameter values of $V$ under some specified conditions. The basic condition is that the model describing the scattering function $V$ is close to the ray model, i. e., to the generalized specular reflection defined by the expression [2]:

$$
V\left(\vec{u}, \vec{u}^{\prime}\right)=\delta\left(\vec{u}^{\prime}-\vec{u}_{*}(\vec{u})\right) .
$$

Here, $\delta$ is the Dirac delta function and $\vec{u}_{*}$ is some specified velocity of reflected from the surface gas atoms that is, in general, different from the specular velocity. The momentum exchange coefficients for the ray scattering function $V$ are expressed by

$$
p=2 \cos \theta\left(\cos \theta+u^{\prime} \cos \theta^{\prime}\right) ; \quad \tau=2 \cos \theta\left(\sin \theta-u^{\prime} \sin \theta^{\prime}\right)
$$

where $u$ and $u^{\prime}$ are the absolute values of the velocities $\vec{u}$ and $\vec{u}^{\prime}$; and $\theta$ and $\theta^{\prime}$ are the corresponding angles for incident and reflected gas atoms [4].

The ray model has a better experimental confirmation (especially combined with diffuse scattering) in comparison with other surface interaction models widely applied in practical DSMC calculations, in particular with the speculardiffuse model [4]. The approximation of real gas-surface characteristics by the ray model is good enough for different physical conditions. Therefore, it can be assumed that the ray model is valid also in the considered flows.

\section{ANALYTICAL MODELS OF DYNAMIC SYSTEM}

Simulating successive gas atoms reflections from channel walls, let us obtain the nonlinear dynamic system; the subscript $m$ indicates the number of reflections (Fig. 1). Denoting $x_{m}=\tan \theta_{m}, x_{m+1}=\tan \theta_{m+1}\left(x_{m}\right.$ and $x_{m+1}$ are the successive values defining the trajectory of a gas particle in the channel) and 

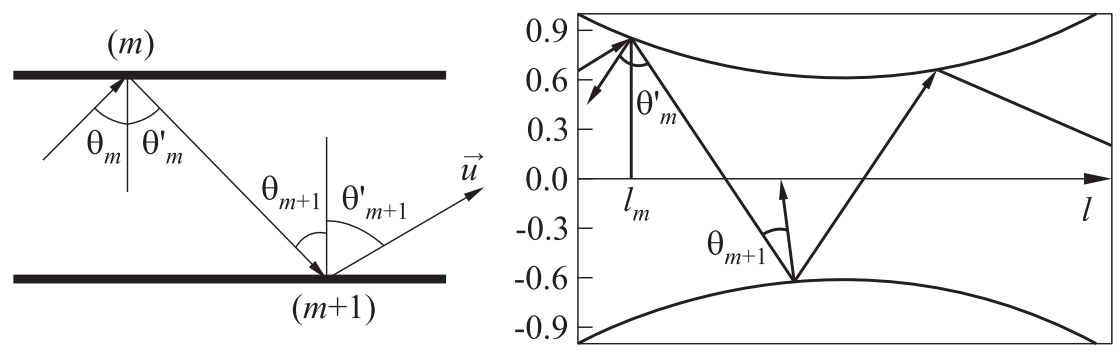

Figure 1 The iterative scheme of successive reflections of a gas atom from the walls in a flat channel $(a)$ or in a nozzle $(b)$

expressing $\theta^{\prime}$ from the presented formulae of momentum exchange coefficients $p$ and $\tau$, one obtains the iterative equation:

$$
x_{m+1}=\tan \left[\psi\left(l_{m}, \arctan \frac{2 x_{m}-\tau\left(1+x_{m}^{2}\right)}{p\left(1+x_{m}^{2}\right)-2}\right)\right] .
$$

Here, the function $\psi$ and the variable $l_{m}$ are determined by the geometrical shape of the channel, and the momentum exchange coefficients $p$ and $\tau$ are assigned by local approximations.

If the solutions become unstable, the nonlinear dynamic system corresponding to Eq. (1) gets many different limit solutions - attractors [1-8]. The corresponding parameters of the scattering function $V$ represent the values of singularity. So, small regions of parameter values exist where the Feigenbaum period-doubling cascade $[4,5,8]$ can be observed. Numerical calculations of the trajectories of gas atoms in a channel demonstrate significant changes of the aerodynamic characteristics of the flow near these values of system parameters. The investigation of the behavior of the nonlinear dynamic system in these regions is very difficult because the corresponding iterative Eq. (1) is much more complicated than the well-known equation for logistic map $x_{m+1}=r x_{m}\left(1-x_{m}\right)$.

Therefore, additional restrictions are taken into account. First, let us consider (following [4-8]) two-dimensional flow, i. e., flat or cylindrical channel (see Fig. 1); then, the function $\psi$ in Eq. (1) is identical, and the connection between the angles $\theta_{m}^{\prime}$ and $\theta_{m+1}$ in successive $m$ th and $(m+1)$ th points of collisions of a gas atom with the surface becomes simple: $\theta_{m}^{\prime}=\theta_{m+1}$. Second, only three of the following most applied local approximations are examined.

1. Depending on three-parameter approximation $[4-7,9]$ based on the expansion of $p$ and $\tau / \sin \theta$ in terms of $\cos ^{n} \theta, n=1,2, \ldots$, this model has been confirmed by experiments in many papers (cited in [4]) from St. Petersburg 
State University and Central Institute of Aviation Motors (Moscow) and can be described by equations:

$$
p=p_{1} \cos \theta+p_{2} \cos ^{2} \theta ; \quad \tau=\tau_{0} \sin \theta \cos \theta .
$$

Special cases of this approximation have been examined by Miroshin [1-3]: $p_{2}=\tau_{0}$ ("model A") and $p_{2}=2$ ("model Z"). The coefficients $p_{1}, p_{2}$, and $\tau_{0}$ can be expressed in terms of aerodynamic values, such as Kn, Mach number, temperature factor, etc. Substitution into Eq. (1) permits to reduce three coefficients $p_{1}, p_{2}$, and $\tau_{0}$ to two parameters $a$ and $b$ transforming the iterative equation (1) to

$$
x_{m+1}=\frac{x_{m}}{a \sqrt{1+x_{m}^{2}}-b}
$$

where

$$
a-\frac{p_{1}}{2-\tau_{0}} ; \quad b=\frac{2-p_{2}}{2-\tau_{0}} .
$$

2. Another approximation suggested also by Miroshin [1] ("model B") reduces three coefficients $p_{2}, p_{4}$, and $\tau_{0}\left(p_{2}\right.$ and $\tau_{0}$ remain the same and $p_{4}$ corresponds to higher power $\cos ^{4} \theta$ ) to two parameters $a_{1}$ and $b_{1}$ transforming the iterative equation (1) to

$$
x_{m+1}=\frac{x_{m}\left[1-a_{1}+\left(1-b_{1}\right) x_{m}^{2}\right]}{a_{1}+b_{1} x_{m}^{2}}
$$

where

$$
a_{1}=\frac{p_{2}+p_{4}-2}{p_{2}-\tau_{0}} ; \quad b_{1}=\frac{p_{2}-2}{p_{2}-\tau_{0}} .
$$

3. A more general approximation containing four coefficients $p_{1}, p_{2}, \tau_{0}$, and $\tau_{1}$ and not yet examined for nonlinear dynamic system parameters corresponds to the iterative equation with the same parameters $a$ and $b$ like in Eq. (2) and a new parameter $d$ :

$$
x_{m+1}=\frac{x_{m}}{a \sqrt{1+x_{m}^{2}}-b}\left(1-\frac{d}{1+x_{m}^{2}}\right)
$$

where

$$
d=\frac{\tau_{1}}{2-\tau_{0}}
$$

For $d=0$, Eq. (4) coincides with Eq. (2). Hence, the first approximation (2) is particular case of the third (4). However, the second model (3) cannot be 
represented in the form (4), but the main properties are similar to the corresponding values of the parameters. Figure 2 illustrates the behavior of the functions (2)-(4) for the values of variables $a, b, c$, and $d$ (and corresponding momentum exchange coefficients) observed in real physical conditions of gassurface interaction (and in the experiments). In particular, $a=2$ and $b=1.7$ for the approximation (2) (dashed line), $a=0.2$ and $b=0.9$ for (3) (dotted line), and $a=2, b=1.8$, and $d=0.3$ for (4) (solid line).

They have similar graphs functions (2)-(4) and are all unimodal: they have single maximum and are monotone on both sides of this maximum. This property is necessary for the initiation of Feigenbaum cascade of bifurcations under the following additional condition: the Schwartz derivative must be negative [4].

Investigating the limit behavior of

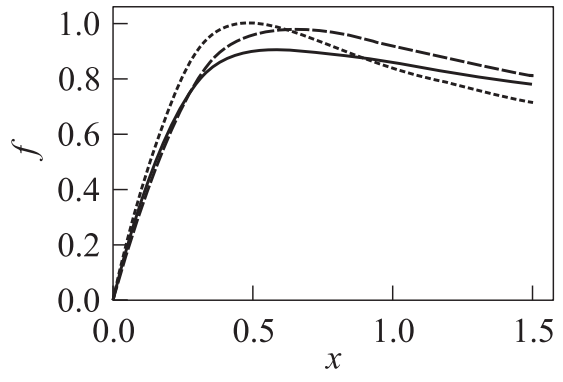

Figure 2 The functions in iterative equations (2)-(4) corresponding to successive reflections of a gas atom from the walls in a channel nonlinear dynamic system by $m \rightarrow \infty$ is the main problem related to studying the stability of rarefied gas flow in a channel [1-7]. The necessary conditions for the existence of Feigenbaum cascade of bifurcation can be proven analytically for iterative equations (2)-(4). All three functions are unimodal (for the mentioned values of the parameters as shown in Fig. 2). This comes from studying the sign of the first derivatives in a very large region of the variables $a, b$, and $d$. Schwartz derivative is negative:

$$
\operatorname{Sf}(x)=\frac{f^{\prime \prime \prime}(x)}{f^{\prime}(x)}-\frac{3}{2}\left(\frac{f^{\prime \prime}(x)}{f^{\prime}(x)}\right)<0
$$

for all functions $f\left(x_{m}\right)$ on the right in the iterative equations (2)-(4). Miroshin has derived it analytically $[1,4]$ for Eq. (3), i.e., for $f_{2}(x)=x\left(1-a_{1}+(1\right.$ $\left.-b_{1}\right) x^{2} /\left(a_{1}+b_{1} x^{2}\right)$, on the base of the representation of Schwartz derivative which can be written after the transformation as

$$
\mathrm{Sf}_{2}(x)=-6 \frac{(b-a)\left(b(1-b) x^{4}-6 a(1-b) x^{2}+a(1-a)\right)}{\left(b(1-b) x^{4}+(3 a-2 a b-b) x^{2}+a(1-a)\right)^{2}} .
$$

A similar representation and the proof of the inequality $\operatorname{Sf}_{1}(x)<0$ is obtained for the iterative equation (2), i. e., for $f_{1}(x)=x /\left(a \sqrt{1+x^{2}}-b\right)$ [4-7], expressing Schwartz derivative as

$$
\operatorname{Sf}_{1}(x)=-3 \frac{\left(a \sqrt{1+x^{2}}-b\right)^{2}+a^{2}-b^{2}}{2\left(1+x^{2}\right)^{2}\left(a-b \sqrt{1+x^{2}}\right)^{2}}
$$


The third model (4), i. e.,

$$
f_{3}(x)=\frac{x}{a \sqrt{1+x^{2}}-b}\left(1-\frac{d}{1+x^{2}}\right),
$$

does not allow a simple formula for Schwartz derivative like (5); it is much more complicated. However, the inequality $\mathrm{Sf}_{3}(x)<0$ can be proved analytically for $a>b,(d(3 a-b)-a)(d-1)>0$, and $x \in[0, \infty)$. For instance, the value for $x=0$ is

$$
\mathrm{Sf}_{3}(0)=-3\left(\frac{a}{a-b}+\frac{2 d}{d-1}\right) .
$$

Negative Schwartz derivative is necessary but not sufficient for the initiation of Feigenbaum cascade of bifurcations; a numerical confirmation is needed. Analytical and numerical investigation of limit solutions demonstrates that all the three iterative equations (2)-(4) can have attractors of different types depending on the parameters $a, b$, and $d$. Limit cycles corresponding to the roots of the equation $f(x)=x$ and stationary point $x=0$ (indicating direct flow along the axis of the channel) are obligatory. Iterative equations (2) and (3) can also have stationary points $x_{1}=\sqrt{(b+1)^{2} / a^{2}-1}, x_{2}=\sqrt{(2 a-1) /(1-2 b)}$, and $x_{0}=\infty$, respectively. The last value has a physical meaning of locking the channel, i. e., its conductivity reduces significantly.

\section{NUMERICAL CALCULATIONS}

The main conclusion from numerical study of the third model (4) is that the attractors and bifurcations in this case are noticeably more various than for the first two models (2) and (3). In particular, up to three attractors exist corresponding to the roots of the equation

$$
a t^{3}-(b+1) t^{2}+d=0 .
$$

The attractors of higher degree could be obtained from the equation $f \cdots f(x)=x$ (where $f(x)$ could be iterated many times) and the set of the solutions could be very rich for some values of $a, b$, and $d$. A typical trajectory of the dynamic system described by the iterative equation (4) is presented in Fig. 3 in coordinates $m$ and $x$ in the region where it is unstable. The dynamic system is discrete; thus, connecting segments are shown only to make the trajectory visible.

Under certain conditions, iterative equations (2)-(4) may have unstable solutions in some regions of the values of gas-surface interaction parameters $a, b$, and $d$. In these regions (which can be found analytically in the described way), comparative small modification of the parameters $a, b$, and $d$ causes significant 


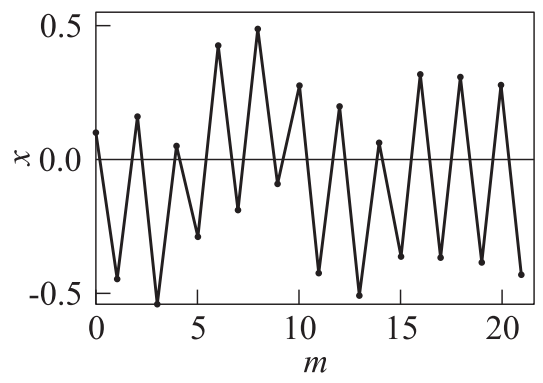

Figure 3 Typical trajectory of the dynamic system (4) for $a=2, b=1.95$, and $d=0.2$ difference between corresponding limit values $x_{m}=\tan \theta_{m}$. From an aerodynamic point of view, it has the following interpretation: macroscopic parameters of the flow may fluctuate unsteady while the difference in microscopic values describing gassurface interaction remains negligible. However, the regions in which the flow becomes unstable are very narrow; therefore, it is difficult to find them numerically or experimentally. In the coordinate system $(a, b)$, the regions of instability obtained analytically are concentrated near the line $a=b$.

A real physical setup of the considered rarefied gas flows has not yet a detailed experimental confirmation because of technical difficulties in performing such experiments.

However, these analytical evaluations are confirmed by numerical calculations.

The best way to study the unstable dynamic systems and their bifurcations is by constructing bifurcation diagrams.

The appearing Feigenbaum period-doubling cascade is shown in three bifurcation diagrams for three considered iterative equations: (2) (Fig. 4a), (3) (Fig. 4b), and (4) (Fig. 4c). Figure 4b, lower frame, illustrates also Lyapunov exponent $\lambda[1-4]$ (parameter $r$ is a combination of $a$ and $b$ in (2)), Fig. $4 a$ is a part of bifurcation diagram for $1.1 \leq a \leq 1.5$, and Fig. $4 c$ is a part of bifurcation diagram corresponding to $1.7 \leq a \leq 2.2$.

The length $l$ of the channel is assumed to be quite large (from 10 to 100) relative to its width in the Monte-Carlo simulations to obtain the discussed nonlinear dynamic effects. Other parameters (local approximation parameters $a$, $b, d$, the part of diffuse scattered gas atoms $\sigma$, and initial angle $\left.\theta_{0}\right)$ are changed in accordance with the known limit analytical solution of iterative equations (2)(4). In the initial section of the channel, a uniform flow is set consisting of $N$ gas atoms having identical velocities with the angle of inclination $\theta_{0}(N$ changes from $10^{4}$ to $5 \cdot 10^{4}$ ).

The ray-diffuse model of scattering of gas atoms is considered with the parameter $a$ (on $x$-axis) and with the geometrical parameter $x=\tan (\theta)$ of the trajectory of a gas atom in the channel on $y$-axis.

The bifurcation diagrams shown in Fig. 4 demonstrate the possible equilibrium (or long-term) values of a system as a function of the bifurcation parameter $a$. Therefore, nonrandom solutions are represented as lines (see Figs. $4 b$ and $4 c$ ) or as black regions (see Figs. $4 a-4 c$ ). However, random solutions can 


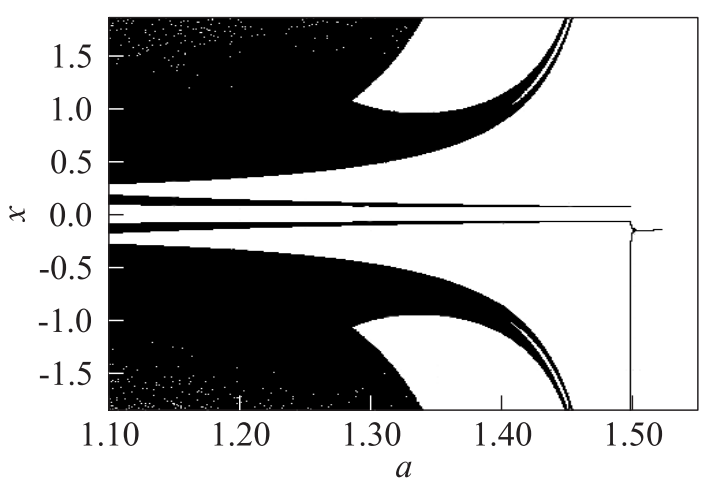

(a)

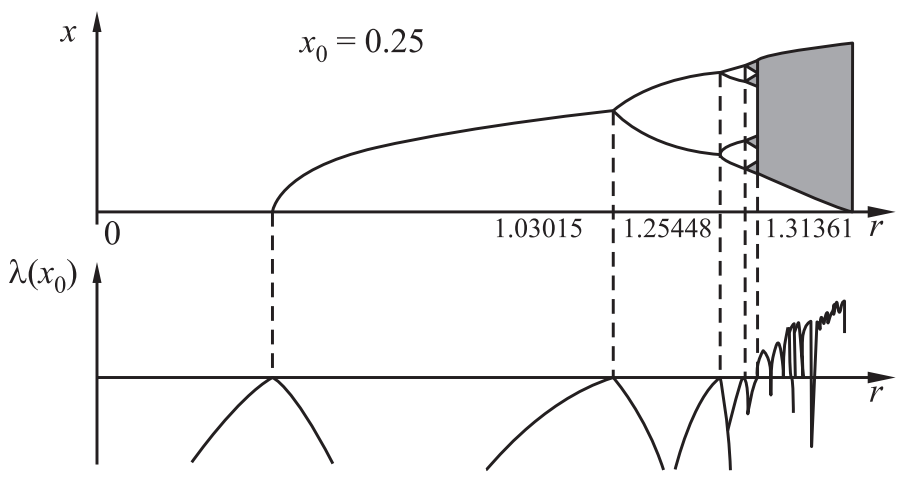

(b)

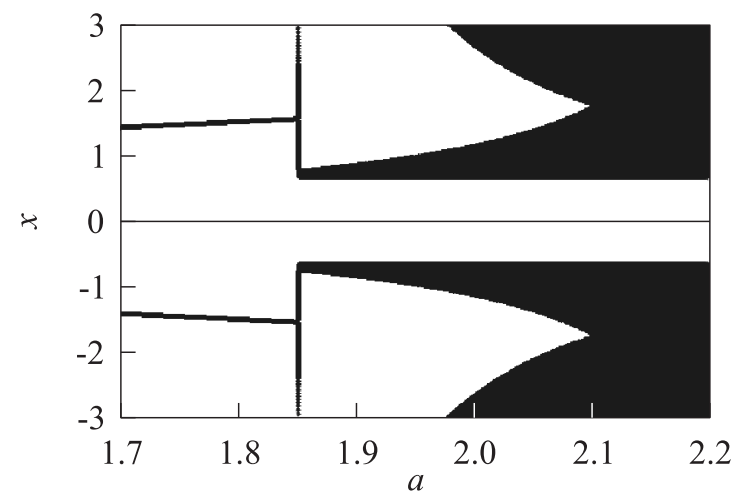

(c)

Figure 4 Bifurcation diagrams for a nonlinear dynamic system corresponding to rarefied gas flow in a channel and iterative equations (2) (a), (3) (b), and (4) (c) 


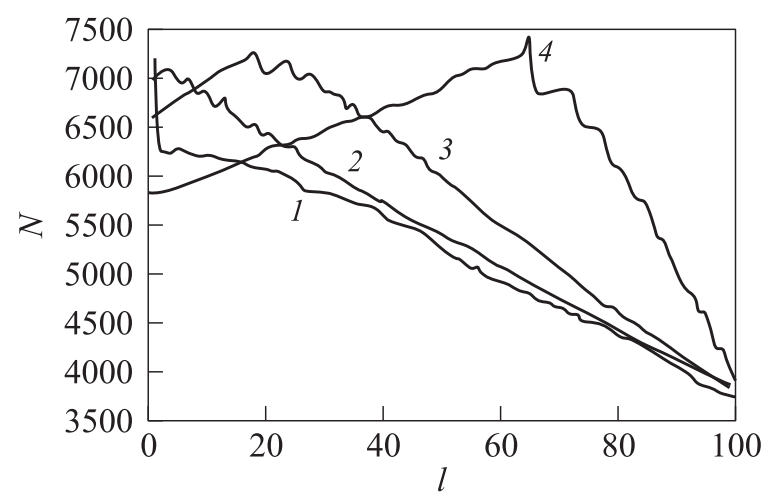

Figure 5 The change of the number $N$ of gas atoms along the channel by the modification of the parameter $a=1.47(1), 1.48$ (2), 1.50 (3), and 1.60 (4) by constant $b=1.7, \sigma=0.05$, and iterative equation (2)

be observed on bifurcation diagrams as distributed sets. At the first sight, the points on these diagrams seem to be distributed randomly but in fact, a sharp structure is well seen in all diagrams. The existence of this structure justifies the approach based on the nonlinear dynamics, and corresponding borders between the regions with different density of the points are the most interesting regions of the instability.

To illustrate the obtained results, comparative graphs of the numerical density of gas atoms in some sections of a channel depending on value $a$ are presented (Fig. 5) for the first model (2).

The scattering function is assumed to be ray-diffuse with the identical value $\sigma=0.1$. The parameters are changed gradually near the points of the instability to demonstrate the variation of the results. For instance, the variable $a$ changes from 1.47 to 1.60 in four steps (see Fig. 5). The largest difference between curves 1 and 2 corresponding to the nearest values $a=1.47$ and 1.48 indicates the region of the instability in the segment $1.47 \leq a \leq 1.48$.

The results of numerical calculations show that for relative small values of $\sigma$, the effect of significant variation of flow parameters by a small modification of gas-surface interaction coefficients $a$ and $b$ is obtained. This effect is qualitatively the same as the effect for the ray scattering $(\sigma=0)$ and it can be observed by the same parameter values of $a$ and $b$.

Near the point of bifurcation, a negligible change of one of the parameters of the ray-diffuse model (less than 1\%) causes also the substantial difference in gas flow in the channel (or in the nozzle). 


\section{CONCLUDING REMARKS}

Monte-Carlo simulation of different nonlinear dynamic systems corresponding to rarefied gas flows in channels has indicated various developments of instability including cascades of period-doubling bifurcations. Several main conclusions follow from the analysis of the comparison:

- adding the extra parameter $d$ allows one extending the region where the dynamic system (related to rarefied gas flow) is unstable in the parametric space. Simulated unstable states of the system are close to physical situations observed in practice;

- numerical calculations confirm the analytical evaluations of the values of the parameters corresponding to unstable rarefied gas flows. More significant in applications is the possibility to use relatively simple analytical representations with the parameters found numerically. Hence, the present study is numerically useful;

- obtained connection between the parameters of the nonlinear dynamic system and momentum exchange coefficients (or accommodation coefficients) makes it possible to express analytic evaluations in terms of aerodynamic characteristics including Knudsen and Mach numbers, temperature factor, etc.; and

- to verify the bifurcations of simulated type experimentally, all considered physical values in the flows are to be set exactly to the same values as detected in the present calculations. Therefore, this problem may be complicated enough.

\section{REFERENCES}

1. Miroshin, R. N. 1994. Bifurkatsii skorosti pri protekanii razrezhennogo gaza v ploskoy shcheli [Bifurcations of the velocity when a rarefied gas flows in a flat gap]. St. Petersburg State University Vestnik Ser. 1. 4(22):72-74.

2. Miroshin, R. N. 1997. O luchevoy modeli vzaimodeystviya atomov razrezhennogo gaza s poverkhnost'yu [On the ray model of the interaction of atoms of a rarefied gas with a surface]. St. Petersburg State University Vestnik Ser. 1. 4(22):74-79.

3. Miroshin, R. N. 1998. Reshenie zadachi o svobodnomolekulyarnom techenii gaza $\mathrm{v}$ polosti klina metodami nelineynoy dinamiki [Solution of the problem of freemolecular flow of gas in the wedge cavity by the methods of nonlinear dynamics]. St. Petersburg State University Vestnik Ser. 1. 1(1):73-77. 
4. Miroshin, R. N., and I. A. Khalidov. 2002. Lokal'nye metody v mekhanike sploshnykh sred [Local methods in continuum mechanics]. St. Petersburg: St. Petersburg State University Publs. 1-202.

5. Aksenova, O. A., and I. A. Khalidov. 2004. Sherokhovatost' poverkhnosti v aerodinamike razrezhennogo gaza: fraktal'nye i statisticheskie modeli [Surface roughness in rarefied gas aerodynamics: Fractal and statistical models]. St. Petersburg: St. Petersburg State University Publs. 120 p.

6. Aksenova, O. A., and I. A. Khalidov. 2011. Influence of rarefied gas-surface interaction on flow stability in channels. AIP Conference Proceedings. Melville, NY: American Institute of Physics. 1333(1):447-451.

7. Aksenova, O.A., and I. A. Khalidov. 2012. Computation of unstable rarefied gas flow in channels with different scattering functions. AIP Conference Proceedings. Melville, NY: American Institute of Physics. 1501:931-937.

8. Aksenova, O. A. 2004. Sopostavlenie fraktal'noy i statisticheskoy modeley sherokhovatosti poverkhnosti $\mathrm{v}$ zadache o rasseyanii atomov razrezhennogo gaza [The comparison of fractal and statistical models of surface roughness in the problem of scattering rarefied gas atoms]. St. Petersburg State University Vestnik Ser. 1. $1(1): 61-66$.

9. Pilipchuk, V.N. 2010. Nonlinear dynamics: Between linear and impact limits. Berlin: Springer. $360 \mathrm{p}$.

10. Barantsev, R. G. 1972. Some problems of gas-solid surface interaction. Prog. Aerosp. Sci. 13:1-80. 